\title{
A Unstructured Nodal Spectral-Element Method for the Navier-Stokes Equations
}

\author{
Lizhen Chen $^{1}$, Jie Shen ${ }^{1,2}$ and Chuanju $\mathrm{Xu}^{1, *}$ \\ ${ }^{1}$ School of Mathematical Sciences, Xiamen University, 361005 Xiamen, China. \\ 2 Department of Mathematics, Purdue University, West Lafayette, IN, 47907, USA.
}

Received 7 January 2011; Accepted (in revised version) 14 July 2011

Communicated by George Karniadakis

Available online 30 January 2012

\begin{abstract}
An unstructured nodal spectral-element method for the Navier-Stokes equations is developed in this paper. The method is based on a triangular and tetrahedral rational approximation and an easy-to-implement nodal basis which fully enjoys the tensorial product property. It allows arbitrary triangular and tetrahedral mesh, affording greater flexibility in handling complex domains while maintaining all essential features of the usual spectral-element method. The details of the implementation and some numerical examples are provided to validate the efficiency and flexibility of the proposed method.
\end{abstract}

AMS subject classifications: 65N35, 65N22, 65F05, 35J05

Key words: Navier-Stokes equations, unstructured mesh, triangular spectral element method.

\section{Introduction}

The spectral-element method combines the geometric flexibility of finite elements with the high accuracy of spectral methods. It exhibits several favorable computational properties, such as the use of tensor products, naturally diagonal mass matrices, and suitability for parallel computation.

However, in order to use the properties of the tensor product, the standard spectralelement method is usually limited to quadrilateral/hexahedral partitions. This requirement makes it difficult to use unstructured mesh for complex geometries. One way to overcome this drawback is to allow the use of triangles/tetrahedrons in the partition. There has been a number of works addressing the so-called triangular spectral methods. The existing spectral methods on triangle can be classified into different types according

*Corresponding author. Email addresses: xunyicao2009@yeah.net (L. Chen), shen7@purdue.edu (J. Shen), cjxu@xmu.edu.cn (C. Xu) 
to the class of functions used in the approximations: (i) approximations by polynomials in triangle through mapping (see, e.g., $[2,5,9,14,19]$ ); (ii) approximations by polynomials in triangle using special nodal points such as Fekete points (see, e.g., $[8,15,16,20]$ ); and (iii) approximations by non-polynomial functions in triangle (see, e.g., $[1,7,18]$ ).

The triangular spectral method based on polynomial spaces were motivated by the classical result that any smooth function can be well approximated by polynomials. Koornwinder [10] and Dubiner [5] constructed the orthogonal polynomials on triangle, often referred as the Dubiner's basis in the spectral-element community. The first practical implementation of the Dubiner basis in the solution of incompressible Navier-Stokes equations was carried out in the spectral-element package NekTar $[9,19]$. Their approach is based on the modal formulation in which the basis functions are Jacobi polynomials with index varying with the polynomial order. A drawback of this modal basis is that there is no corresponding nodal basis, making it more difficult, to implement. Recently, a triangular spectral method using rational polynomials was proposed and analyzed for elliptic problems (cf. [18]). It is extended to the Stokes problem on a triangle in [3]. The main advantage of this method is that a nodal basis is available, so it can be incorporated into the usual nodal spectral-element framework. In particular, it preserves the tensor product structure which enables the fast evaluation of the matrix-vector multiplications.

In this paper, we design an unstructured spectral-element method for the NavierStokes equations. More precisely, we describe in detail how to formulate the nodal basis with two-dimensional unstructured meshes for the Stokes equations, and apply them for solving time dependent Navier-Stokes equations. Our nodal basis functions in triangles/tetrahedrons are constructed from the standard tensor product of Lagrangian polynomials defined on the 2-D Gauss-Lobatto points through the Duffy mapping. The advantages of this nodal basis are that it allows arbitrary mixture of triangular and rectangular elements; enjoys the fully tensorial-product property, and can be easily incorporated into an existing spectral-element code.

The paper is organized as follows. In the next section, we first present the rational spectral-element method for the Stokes equations on a single triangle. In Section 3, we extend the method to 2-D unstructured mesh with arbitrary mixture of triangular and quadrilateral elements. In Section 4, we apply this spectral-element method to the Navier-Stokes equations and present several numerical experiments exhibiting its flexibility and accuracy. Some concluding remarks are given in the final section.

\section{Preliminaries}

In this section, we introduce some necessary notations and recall briefly the triangular spectral method for the Stokes equations developed recently in [3].

Throughout this paper, we use boldface letters to denote vectors and vector functions. Let $c$ be a generic positive constant independent of any functions and of any discretization parameters. We use the expression $A \lesssim B$ to mean that $A \leqslant c B$, and use the expression 
$A \cong B$ to mean that $A \lesssim B \lesssim A$. For a bounded domain $\Omega$ and a generic positive weight function $\omega$, we denote the inner product of $L_{\omega}^{2}(\Omega)$ by

$$
(u, v)_{\omega, \Omega}:=\int_{\Omega} u v \omega d x
$$

and the associated norm by $\|\cdot\|_{\omega, \Omega}$. We also use $H_{\omega}^{m}(\Omega)$ and $H_{0, \omega}^{m}(\Omega)$ to denote the usual weighted Sobolev spaces, whose norm and semi-norm are denoted by $\|u\|_{m, \omega, \Omega}$ and $|u|_{m, \omega, \Omega}$, respectively. We also need the space

$$
L_{0, \omega}^{2}(\Omega)=\left\{\phi \in L_{\omega}^{2}(\Omega): \int_{\Omega} \phi \omega d x=0\right\} .
$$

In cases where no confusion would arise, $\omega$ (if $\omega=1$ particularly) and/or $\Omega$ may be dropped from the notations. We denote by $\mathbb{N}$ the set of all non-negative integers and $\Lambda=(-1,1)$. For any $N \in \mathbb{N}$, we denote by $\mathbb{P}_{N}(\Lambda)$ the set of all polynomials of degree $\leq N$ defined in $\Lambda$, and set $\mathbb{P}_{N}^{0}(\Lambda):=\left\{\phi \in \mathbb{P}_{N}(\Lambda): \phi( \pm 1)=0\right\}$.

Let us denote by $\triangle$ the triangle domain

$$
\triangle=\{(x, y): 0<x, y<1,0<x+y<1\} .
$$

We consider the Stokes equations in $\triangle$ : For a given forcing function $f \in L^{2}(\triangle)^{2}$, find the velocity vector $u$ and the pressure $p$ such that

$$
\begin{cases}-\Delta \boldsymbol{u}+\nabla p=f, & \text { in } \triangle, \\ \nabla \cdot \boldsymbol{u}=0, & \text { in } \triangle, \\ \boldsymbol{u}=\mathbf{0}, & \text { on } \partial \triangle .\end{cases}
$$

\subsection{Duffy mapping}

In order to describe the triangular spectral method for (2.1), we will use two coordinate systems: the Cartesian coordinate $(x, y)$-system for the triangle $\triangle$ and the $(\xi, \eta)$-system for the square $\square:=\Lambda^{2}$ connected by the Duffy mapping $\boldsymbol{x}=\boldsymbol{F}(\boldsymbol{\xi})(\boldsymbol{x}=(x, y)$ and $\boldsymbol{\xi}=(\boldsymbol{\xi}, \eta))$ :

$$
x=\frac{1}{4}(1+\xi)(1-\eta), \quad y=\frac{1+\eta}{2}, \quad \forall(\xi, \eta) \in \square,
$$

with its inverse $\boldsymbol{\xi}=\boldsymbol{F}^{-1}(\boldsymbol{x})$ from $\triangle$ to $\square$ by

$$
\xi=\frac{2 x}{1-y}-1, \quad \eta=2 y-1, \quad \forall(x, y) \in \triangle .
$$

Note that the mapping (2.2) is singular as it maps the top side of $\square$ to the single vertex $(0,1)$ of $\triangle$. 
(a)

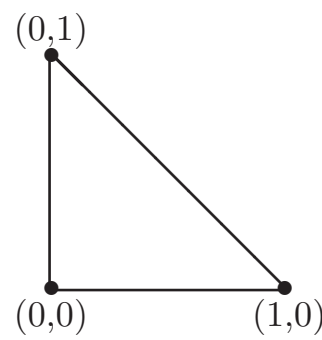

(b)

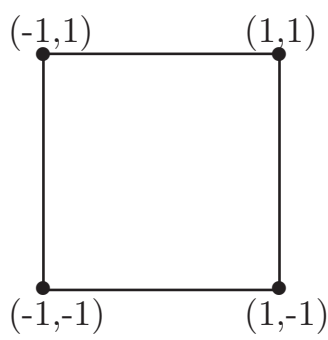

Figure 1: (a) Domain $\triangle$ with coordinate x. (b) Domain $\square$ with coordinate $\xi$.

We collect below some properties of Duffy map, which will be used in the sequel:

$$
\begin{aligned}
& \frac{\partial \xi}{\partial x}=\frac{2}{1-y}=\frac{4}{1-\eta}, \quad \frac{\partial \xi}{\partial y}=\frac{2 x}{(1-y)^{2}}=\frac{2(1+\xi)}{1-\eta}, \quad \frac{\partial \eta}{\partial x}=0, \quad \frac{\partial \eta}{\partial y}=2, \\
& \frac{\partial x}{\partial \xi}=\frac{1-y}{2}=\frac{1-\eta}{4}, \quad \frac{\partial x}{\partial \eta}=\frac{x}{2(1-y)}=-\frac{1+\xi}{4}, \quad \frac{\partial y}{\partial \xi}=0, \quad \frac{\partial y}{\partial \eta}=\frac{1}{2} .
\end{aligned}
$$

From the above, one easily finds the determinant of the Jacobian for (2.2):

$$
\operatorname{det}\left(\frac{\partial(x, y)}{\partial(\xi, \eta)}\right)=\frac{1-\eta}{8}=\frac{1-y}{4} .
$$

Throughout the paper, we shall associate a function $u$ in $\triangle$ with a function $\widetilde{u}$ in $\square$ through

$$
\widetilde{u}(\xi, \eta)=u(x, y), \quad x=\frac{1}{4}(1+\xi)(1-\eta), \quad y=\frac{1+\eta}{2}, \quad \forall(\xi, \eta) \in \square .
$$

Then we have

$$
\nabla_{x} u=\left(\partial_{x} u, \partial_{y} u\right)^{T}=\left(\frac{4}{1-\eta} \partial_{\tilde{\xi}} \widetilde{u}, \frac{2(1+\tilde{\xi})}{1-\eta} \partial_{\tilde{\xi}} \widetilde{u}+2 \partial_{\eta} \widetilde{u}\right)^{T},
$$

and inversely,

$$
\nabla_{\xi \widetilde{u}}=\left(\partial_{\tilde{\xi}} \widetilde{u}, \partial_{\eta} \widetilde{u}\right)^{T}=\left(\frac{1-y}{2} \partial_{x} u, \frac{x}{2(1-y)} \partial_{x} u+\frac{1}{2} \partial_{y} u\right)^{T} .
$$

From (2.7), we have immediately

$$
\partial_{\tilde{\xi}} \widetilde{u}(\widetilde{\zeta}, 1)=0 \text { a.e. if } \partial_{x} u \text { is a measurable function. }
$$

In other words, if the approximation solution is chosen such that its partial derivative with respect to $x$ is bounded in $\triangle$, then its transformation in $\square$ by Duffy mapping must be constant at the top side. This fact should be kept in mind in constructing the basis functions for the approximation space. 


\subsection{Rational approximation in triangle}

The approximation space to be used in our method consists of the rational functions generated by polynomials in the reference square through the Duffy transform. Precisely, let $\tilde{\mathcal{R}}_{m n}(\xi, \eta)$ be the polynomial in $\square$ defined by:

$$
\tilde{\mathcal{R}}_{m n}(\xi, \eta)=J_{m}^{0,0}(\xi) J_{n}^{1,0}(\eta), \quad \forall(\xi, \eta) \in \square,
$$

where $J_{k}^{\alpha, \beta}(\zeta), \zeta \in \Lambda$, is the classical Jacobi polynomial of degree $k$. Then we define the rational function $\mathcal{R}_{m n}(x, y)$ in $\triangle$ by the Duffy transformation of $\tilde{\mathcal{R}}_{m n}(\xi, \eta)$, i.e.,

$$
\mathcal{R}_{m n}(x, y)=\tilde{\mathcal{R}}_{m n}\left(\frac{2 x}{1-y}-1,2 y-1\right)=J_{m}^{0,0}\left(\frac{2 x}{1-y}-1\right) J_{n}^{1,0}(2 y-1), \quad \forall(x, y) \in \triangle,
$$

and the approximation spaces and their transformations as follows:

$$
\begin{aligned}
& \mathbb{Q}_{N}(\triangle)=\operatorname{span}\left\{\mathcal{R}_{m n}(x, y), 0 \leq m, n \leq N,(x, y) \in \triangle\right\}, \\
& \widetilde{\mathbb{Q}}_{N}(\square)=\operatorname{span}\left\{\widetilde{\mathcal{R}}_{m n}(\xi, \eta), 0 \leq m, n \leq N,(\xi, \eta) \in \square\right\}, \\
& \mathbb{Q}_{N}^{0}(\triangle)=\left\{v \in \mathbb{Q}_{N}(\triangle),\left.v\right|_{\partial \triangle}=0\right\}, \\
& \widetilde{\mathbb{Q}}_{N}^{0}(\square)=\left\{v \in \widetilde{\mathbb{Q}}_{N}(\square),\left.v\right|_{\partial \square}=0\right\} .
\end{aligned}
$$

By the properties of the Jacobi polynomials, we have the following orthogonality:

$$
\begin{aligned}
& \int_{\triangle} \mathcal{R}_{m n}(x, y) \mathcal{R}_{m^{\prime} n^{\prime}}(x, y) d x d y \\
= & \frac{1}{8} \int_{-1}^{1} J_{m}^{0,0}(\xi) J_{m^{\prime}}^{0,0}(\xi) d \xi \int_{-1}^{1} J_{n}^{1,0}(\eta) J_{n^{\prime}}^{1,0}(\eta)(1-\eta) d \eta \\
= & \gamma_{m n} \delta_{m m^{\prime}} \delta_{n n^{\prime}}, \quad \text { with } \gamma_{m n}=\frac{1}{2(n+1)(2 m+1)} .
\end{aligned}
$$

Therefore, any function $u \in L^{2}(\triangle)$ has the expression

$$
u(x, y)=\sum_{m=0}^{\infty} \sum_{n=0}^{\infty} \widehat{u}_{m n} \mathcal{R}_{m n}(x, y),
$$

with the coefficient $\widehat{u}_{m n}$ given by

$$
\widehat{u}_{m n}=\frac{1}{\gamma_{m n}} \int_{\triangle} u(x, y) \mathcal{R}_{m n}(x, y) d x d y .
$$

On the other hand, we have $\widetilde{u} \in L_{\omega}^{2}(\square)$ if and only if $u \in L^{2}(\triangle)$, where the weight function

$$
\omega:=\frac{1-\eta}{8}
$$


is the Jacobian in (2.6), and

$$
\widetilde{u}(\xi, \eta)=\sum_{m=0}^{\infty} \sum_{n=0}^{\infty} \widehat{u}_{m n} \widetilde{\mathcal{R}}_{m n}(\xi, \eta),
$$

with $\widehat{u}_{m n}$ given in (2.12), or expressed in the alternative form:

$$
\widehat{u}_{m n}=\frac{1}{\gamma_{m n}} \int_{\square} \widetilde{u}(\xi, \eta) \widetilde{\mathcal{R}}_{m n}(\xi, \eta) \omega d \xi d \eta .
$$

The inner product on $L^{2}(\triangle)$ is defined as

$$
(\phi, \psi)=\int_{\triangle} \phi(x, y) \psi(x, y) d x d y=\int_{\square} \widetilde{\phi}(\xi, \eta) \widetilde{\psi}(\xi, \eta) \frac{1-\eta}{8} d \xi d \eta, \quad \forall \phi, \psi \in C(\triangle),
$$

where $(1-\eta) / 8$ is the Jacobian given in (2.6).

Let $\xi_{p}, p=0,1, \cdots, N$, be the Legendre-Gauss-Lobatto points associated to $L_{N}$, i.e., zeros of $\left(1-z^{2}\right) L_{N}^{\prime}(z)$, and $\omega_{p}, p=0,1, \cdots, N$, be the corresponding weights. We can then define the discrete inner product $(\cdot, \cdot)_{N}$ on $\triangle$ :

$$
(\phi, \psi)_{N}=\sum_{p, q=0}^{N} \widetilde{\phi}\left(\xi_{p}, \xi_{q}\right) \widetilde{\psi}\left(\xi_{p}, \xi_{q}\right) \frac{1-\xi_{q}}{8} \omega_{p} \omega_{q}, \quad \forall \phi, \psi \in C(\triangle) .
$$

\subsection{Triangular spectral method for the Stokes equations}

Now we are in a position to define our triangular spectral method for (2.1). Let us denote $X=H_{0}^{1}(\triangle), M=L_{0}^{2}(\triangle)$ and $\boldsymbol{X}=X^{2}$. We introduce the bilinear form $a(\cdot, \cdot)$ over $\boldsymbol{X} \times \boldsymbol{X}$ by

$$
a(\boldsymbol{v}, \boldsymbol{w})=\int_{\triangle} \nabla \boldsymbol{v} \nabla \boldsymbol{w} d x d y, \quad \forall \boldsymbol{v}, \boldsymbol{w} \in X
$$

and the bilinear form $b(\cdot, \cdot)$ over $\boldsymbol{X} \times M$ by,

$$
b(v, q)=-\int_{\triangle} \nabla \cdot v q d x d y, \quad \forall v \in X, \quad q \in M .
$$

Then, the weak form of (2.1) reads: Find $\boldsymbol{u} \in \boldsymbol{X}$ and $p \in M$ such that

$$
\begin{cases}a(\boldsymbol{u}, \boldsymbol{v})+b(\boldsymbol{v}, p)=(\boldsymbol{f}, \boldsymbol{v}), & \forall \boldsymbol{v} \in \boldsymbol{X} \\ b(\boldsymbol{u}, q)=0, & \forall q \in M .\end{cases}
$$

Let us define the rational approximation spaces

$$
X_{N}=X \cap Q_{N}(\triangle), \quad M_{N}=M \cap Q_{N-2}(\triangle), \quad X_{N}=X_{N}^{2},
$$


and the discrete bilinear forms

$$
a_{N}\left(\boldsymbol{u}_{N}, \boldsymbol{v}_{N}\right)=\left(\nabla \boldsymbol{u}_{N}, \nabla \boldsymbol{v}_{N}\right)_{N}, \quad b_{N}\left(\boldsymbol{v}_{N}, q_{N}\right)=-\left(q_{N}, \nabla \cdot \boldsymbol{v}_{N}\right)_{N} .
$$

Then, our triangular spectral method for (2.15) is as follows: Find $\boldsymbol{u}_{N} \in \boldsymbol{X}_{N}, p_{N} \in M_{N}$, such that

$$
\begin{cases}a_{N}\left(\boldsymbol{u}_{N}, \boldsymbol{v}_{N}\right)+b_{N}\left(\boldsymbol{v}_{N}, p_{N}\right)=\left(\boldsymbol{f}, \boldsymbol{v}_{N}\right), & \forall \boldsymbol{v}_{N} \in \boldsymbol{X}_{N} \\ b_{N}\left(\boldsymbol{u}_{N}, q_{N}\right)=0, & \forall q_{N} \in M_{N} .\end{cases}
$$

The well-posedness of the discrete problem (2.18) as well as its error analysis was established in [3].

\subsection{A nodal basis}

Under the Duffy mapping, $X_{N}, M_{N}$ are transformed into:

$$
\widetilde{X}_{N}:=\left\{\varphi \in \widetilde{\mathbb{Q}}_{N}^{0}(\square), \partial_{\xi} \varphi(\xi, 1)=0\right\}, \quad \widetilde{M}_{N}:=\widetilde{\mathbb{Q}}_{N-2}(\square) \cap L_{0, \omega}^{2}(\square),
$$

where $\boldsymbol{\omega}$ is defined in (2.13) and $\widetilde{\mathrm{Q}}_{N}(\square)$ is given in (2.11).

Let $h_{p}(\xi), p=0,1, \cdots, N$, are the one-dimensional Lagrangian interpolants associated with the Legendre-Gauss-Lobatto points, such that $h_{p}(\xi) \in \boldsymbol{P}_{N}(\Lambda), h_{p}\left(\xi_{q}\right)=\delta_{p q}, 0 \leq p, q \leq N$. Let $l_{p}(\xi), p=1, \cdots, N-1$, are the one-dimensional Lagrangian interpolants associated with the $N-1$ interior Legendre-Gauss-Lobatto points, such that $l_{p}(\xi) \in \boldsymbol{P}_{N-2}(\Lambda), l_{p}\left(\xi_{q}\right)=\delta_{p q}$, $1 \leq p, q \leq N-1$. Then,

$$
\begin{aligned}
& \widetilde{\mathbb{Q}}_{N}(\square)=\operatorname{span}\left\{h_{i}(\xi) h_{j}(\eta), 0 \leq i, j \leq N\right\}, \\
& \widetilde{\mathbb{Q}}_{N}^{0}(\square)=\operatorname{span}\left\{h_{i}(\xi) h_{j}(\eta), 1 \leq i, j \leq N-1\right\}, \\
& \widetilde{X}_{N}=\widetilde{\mathbb{Q}}_{N}^{0}(\square), \\
& \widetilde{M}_{N}=\operatorname{span}\left\{l_{i}(\xi) l_{j}(\eta), 1 \leq i, j \leq N-1\right\} \cap L_{0, \omega}^{2}(\square) .
\end{aligned}
$$

Let

$$
\widetilde{\varphi}_{i j}(\xi, \eta)=h_{i}(\xi) h_{j}(\eta), \quad \widetilde{\psi}_{i j}(\xi, \eta)=l_{i}(\xi) l_{j}(\eta),
$$

and define the basis functions

$$
\varphi_{i j}(x, y)=\widetilde{\varphi}_{i j}(\xi, \eta), \quad \psi_{i j}(x, y)=\widetilde{\psi}_{i j}(\xi, \eta)
$$

through the Duffy mapping, then we have

$$
\begin{aligned}
& \boldsymbol{X}_{N}=\operatorname{span}\left\{\left(\varphi_{i j}(x, y), 0\right),\left(0, \varphi_{i j}(x, y)\right), 1 \leq i, j \leq N-1\right\}, \\
& M_{N}=\operatorname{span}\left\{\psi_{i j}(x, y), 1 \leq i, j \leq N-1\right\} \cap L_{0}^{2}(\triangle) .
\end{aligned}
$$



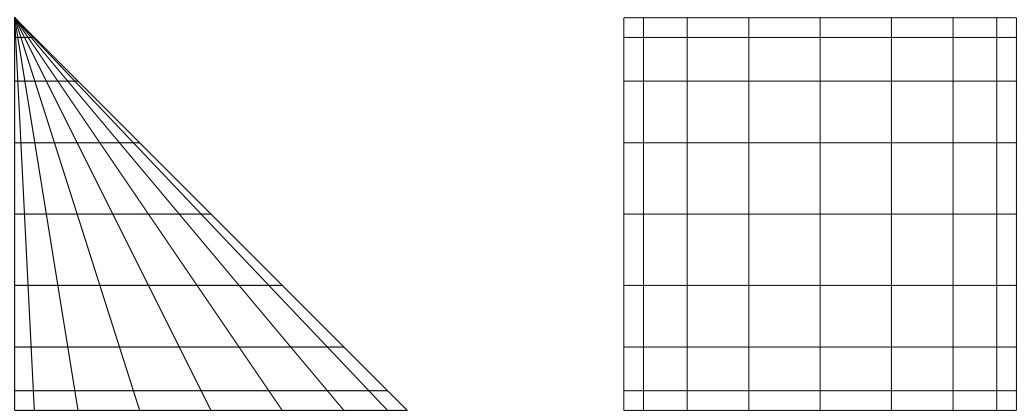

Figure 2: Mapping of the triangle to square domain.

We can then express $\boldsymbol{u}_{N}$ and $p_{N}$, solution of (2.18), as follows:

$$
\begin{aligned}
& \boldsymbol{u}_{N}(x, y)=\widetilde{\boldsymbol{u}}_{N}(\xi, \eta)=\sum_{i, j=1}^{N-1} \boldsymbol{u}_{i j} \widetilde{\varphi}_{i j}(\xi, \eta), \\
& p_{N}(x, y)=\widetilde{p}_{N}(\xi, \eta)=\sum_{i, j=1}^{N-1} p_{i j} \widetilde{\psi}_{i j}(\xi, \eta),
\end{aligned}
$$

where, by definition of the basis functions,

$$
\boldsymbol{u}_{i j}=\widetilde{\boldsymbol{u}}_{N}\left(\xi_{i}, \xi_{j}\right)=\boldsymbol{u}_{N}\left(x_{i}, y_{j}\right), \quad p_{i j}=\widetilde{p}_{N}\left(\xi_{i}, \xi_{j}\right)=p_{N}\left(x_{i}, y_{j}\right), \quad 1 \leq i, j \leq N-1,
$$

with $\left(x_{i}, y_{j}\right)$ the mapped points in $\triangle$ of the Gauss-Lobatto points $\left(\xi_{i}, \xi_{j}\right)$, i.e., $\left(x_{i}, y_{j}\right)=$ $\boldsymbol{F}\left(\xi_{i}, \xi_{j}\right)$; see Fig. 2.

Plugging the above expansions into (2.18), we arrive at the following matrix system:

$$
\left\{\begin{array}{l}
A_{N} \underline{\boldsymbol{u}}+\boldsymbol{D}_{N} \underline{p}=\boldsymbol{B}_{N} \underline{f}, \\
\boldsymbol{D}_{N}^{T} \underline{\boldsymbol{u}}=0,
\end{array}\right.
$$

where $f$ is a vector representation of $f$ at the Gauss-Lobatto points. The matrices $A_{N}$, $\boldsymbol{D}_{N}$, and $\boldsymbol{B}_{N}$ are block-diagonal matrices with 2 blocks each. The blocks of $\boldsymbol{A}_{N}$ are the discrete Laplace operator, and those of $\boldsymbol{D}_{N}$ are associated to the different components of the discrete gradient operators, while blocks of $\boldsymbol{B}_{N}$ are the mass matrices.

\section{Unstructured nodal spectral-element method}

We now provide some details on integrating the triangular spectral method for the Stokes equations presented in the last section into the general spectral-element framework. This integration will enable us to use an arbitrary mixture of triangular and quadrilateral elements in a traditional spectral-element code. 


\subsection{Spectral-element formulation with unstructured mesh}

Let $\Omega$ be a polygonal domain, which is decomposed into a number of elements:

$$
\bar{\Omega}=\bigcup_{k=1}^{K} \bar{\Omega}_{k}, \quad \Omega_{i} \cap \Omega_{j}=\varnothing, \quad i \neq j ; \quad \text { each } \Omega_{i} \text { is a triangle or a quadrilateral. }
$$

We assume that the decomposition is conforming in the sense that the intersection $\bar{\Omega}_{k} \cap \bar{\Omega}_{j}$ $(1 \leq k \leq j \leq K)$ is either empty or a vertex or a whole side of $\Omega_{k}$ and $\Omega_{j}$.

Let $\boldsymbol{F}_{k}$ be the mapping which maps $\square$ onto $\bar{\Omega}_{k}$. If $\Omega_{k}$ is a triangle, the mapping is given in (A.1) below; while if $\Omega_{k}$ is a quadrilateral, we use the usual bilinear blended mapping (cf. [6]).

Remark 3.1. Note that the above partition and mapping are not "spectrally admissible" (cf. [11]) in the usual sense of spectral-element formulation, since the Jacobian of the inverse mapping of $\boldsymbol{F}_{k}$, in the case $\Omega_{k}$ is a triangle, is not bounded. This relaxation on $\boldsymbol{F}_{k}$ allows us to use unstructured mesh with both quadrilaterals and triangles while maintaining the spectral accuracy.

We now define the local approximation space and spectral-element spaces as follows:

$$
\begin{aligned}
& \mathbb{Q}_{N}^{k}=\left\{v_{N} \in L^{2}\left(\Omega_{k}\right), v_{N} \circ \boldsymbol{F}_{k} \in \widetilde{\mathbb{Q}}_{N}(\square)\right\}, \quad 1 \leq k \leq K, \\
& X_{N}^{k}=\mathbb{Q}_{N}^{k} \cap H^{1}\left(\Omega_{k}\right), \\
& X_{N}=\left\{v_{N} \in H_{0}^{1}(\Omega),\left.v_{N}\right|_{\Omega_{k}} \in X_{N}^{k}, 1 \leq k \leq K\right\} \\
& M_{N}=\left\{q_{N} \in L_{0}^{2}(\Omega),\left.q_{N}\right|_{\Omega_{k}} \in \mathbb{Q}_{N-2}^{k}, 1 \leq k \leq K\right\} .
\end{aligned}
$$

It is readily seen that the local space $\mathbb{Q}_{N}^{k}$ contains rational functions if $\Omega_{k}$ is a triangle, while it reduces to classical polynomial space if $\Omega_{k}$ is a quadrilateral.

With the above preparation, the unstructured nodal spectral-element approximation to the Stokes equations on $\Omega$ is as follows: Find $\boldsymbol{u}_{N} \in \boldsymbol{X}_{N}, p_{N} \in M_{N}$ such that

$$
\begin{cases}\sum_{k=1}^{K}\left(\nabla \boldsymbol{u}_{N}, \nabla \boldsymbol{v}_{N}\right)_{N, \Omega_{k}}-\sum_{k=1}^{K}\left(p_{N}, \nabla \cdot \boldsymbol{v}_{N}\right)_{N, \Omega_{k}}=\sum_{k=1}^{K}\left(\boldsymbol{f}, \boldsymbol{v}_{N}\right)_{N, \Omega_{k}}, & \forall \boldsymbol{v}_{N} \in \boldsymbol{X}_{N}, \\ \sum_{k=1}^{K}\left(q_{N}, \nabla \cdot \boldsymbol{u}_{N}\right)_{N, \Omega_{k}}=0, & \forall q_{N} \in M_{N},\end{cases}
$$

where

$$
(\phi, \psi)_{N, \Omega_{k}}=\sum_{p, q=0}^{N} \phi \circ \boldsymbol{F}_{k}\left(\xi_{p}, \xi_{q}\right) \psi \circ \boldsymbol{F}_{k}\left(\xi_{p}, \xi_{q}\right) J_{k} \omega_{p} \omega_{q}
$$

with $J_{k}$ being Jacobian of the mapping $\boldsymbol{F}_{k}$. 


\subsection{Implementation details}

We now describe the implementation details of our unstructured spectral method (3.2) based on a simple nodal basis for the velocity and pressure. More precisely, we describe below the formation of the linear system corresponding to (3.2) and the fast matrix-vector multiplications.

Let us describe how to deal with the term $\left(\nabla \boldsymbol{u}_{N}, \nabla \boldsymbol{v}_{N}\right)_{N, \Omega_{k}}$ in detail. Other terms can be treated in a similar fashion. By definition, we have

$$
\begin{aligned}
\left(\nabla \boldsymbol{u}_{N}, \nabla \boldsymbol{v}_{N}\right)_{N, \Omega_{k}}= & \sum_{p, q=0}^{N} G_{1}^{k}\left(\xi_{p}, \xi_{q}\right) \frac{\partial \widetilde{\boldsymbol{u}}_{N}^{k}}{\partial \xi^{2}} \frac{\partial \widetilde{\boldsymbol{v}}_{N}^{k}}{\partial \xi^{\prime}}\left(\xi_{p}, \xi_{q}\right) \omega_{p} \omega_{q} \\
& +\sum_{p, q=0}^{N} G_{2}^{k}\left(\xi_{p}, \xi_{q}\right) \frac{\partial \widetilde{\boldsymbol{u}}_{N}^{k}}{\partial \eta} \frac{\partial \widetilde{\boldsymbol{v}}_{N}^{k}}{\partial \eta}\left(\xi_{p}, \xi_{q}\right) \omega_{p} \omega_{q} \\
& +\sum_{p, q=0}^{N} G_{3}^{k}\left(\xi_{p}, \xi_{q}\right)\left(\frac{\partial \widetilde{\boldsymbol{u}}_{N}^{k}}{\partial \xi} \frac{\partial \widetilde{\boldsymbol{v}}_{N}^{k}}{\partial \eta}+\frac{\partial \widetilde{\boldsymbol{u}}_{N}^{k}}{\partial \eta} \frac{\partial \widetilde{\boldsymbol{v}}_{N}^{k}}{\partial \xi^{\prime}}\right)\left(\xi_{p}, \xi_{q}\right) \omega_{p} \omega_{q},
\end{aligned}
$$

where, for a function $v$ defined in $\Omega_{k}, \widetilde{v}^{k}$ means $v \circ \boldsymbol{F}_{k}$, and $G_{1}^{k}, G_{2}^{k}$, and $G_{3}^{k}$ are defined as

$$
\begin{aligned}
& G_{1}^{k}=\frac{1}{\left|J_{k}\right|}\left[\left(\frac{\partial x}{\partial \eta}\right)^{2}+\left(\frac{\partial y}{\partial \eta}\right)^{2}\right], \\
& G_{2}^{k}=\frac{1}{\left|J_{k}\right|}\left[\left(\frac{\partial x}{\partial \xi}\right)^{2}+\left(\frac{\partial y}{\partial \xi}\right)^{2}\right], \\
& G_{3}^{k}=-\frac{1}{\left|J_{k}\right|}\left[\frac{\partial x}{\partial \xi} \frac{\partial x}{\partial \eta}+\frac{\partial y}{\partial \xi} \frac{\partial y}{\partial \eta}\right] .
\end{aligned}
$$

The evaluation of these geometric factors in the case of triangular elements are given in the Appendix.

To derive the linear system associated to problem (3.2), we need a suitable basis for the spectral-element approximation spaces defined in (3.1). We start by constructing the basis functions for the local spaces associated to a given element $\Omega_{k}$. Since the construction for the quadrilateral elements is standard (cf. $[17,22])$, we shall only consider the case with triangular elements.

In this case, as indicated in (2.9), we see that the transformation of the velocity local space $\mathbb{Q}_{N}^{k} \cap H^{1}\left(\Omega_{k}\right)$ satisfies

$$
X_{N}^{k}=\left\{v_{N} \in H^{1}\left(\Omega_{k}\right), v_{N} \circ \boldsymbol{F}_{k} \in \widetilde{\mathbb{Q}}_{N}(\square), \partial_{\xi}\left(v_{N} \circ \boldsymbol{F}_{k}\right)(\cdot, 1)=0\right\},
$$

where, no loss of generality, we have supposed that the top side of $\square$ is the collapsed side, which is mapped to a vertex of the triangle $\Omega_{k}$ through $\boldsymbol{F}_{k}$. In other words, the transformation in $\square$ of the velocity local space consists of the polynomials of degree $\leq N$, 
which take constant values at the top side. A closer examination shows that the most natural basis for the space $X_{N}^{k}$ is the set of the following rational functions:

$$
\begin{aligned}
X_{N}^{k}=\operatorname{span}\left\{\varphi_{i j}(x, y), \varphi_{0 N}(x, y): \varphi_{i j} \circ \boldsymbol{F}_{k}(\xi, \eta)=h_{i}(\xi) h_{j}(\eta),\right. \\
\left.0 \leq i \leq N, 0 \leq j \leq N-1, \varphi_{0 N} \circ \boldsymbol{F}_{k}(\xi, \eta)=h_{N}(\eta)\right\} .
\end{aligned}
$$

Introducing the index set

$$
\Xi_{N}:=\{(i, j): 0 \leq i \leq N, 0 \leq j \leq N-1\} \cup\{(0, N)\},
$$

we can then write

$$
X_{N}^{k}=\operatorname{span}\left\{\varphi_{i j}(x, y):(i, j) \in \Xi_{N}\right\}
$$

Here, one needs to pay a particular attention to the basis function $\varphi_{i j}$ corresponding to $(i, j)=(0, N)$. This basis function is associated to the vertex which corresponds to the collapsed side in the reference domain $\square$. Therefore all basis functions satisfy the condition:

$$
\partial_{\xi} \widetilde{\varphi}_{i j}(\xi, 1)=0, \quad \forall \xi \in \Lambda, \quad \forall(i, j) \in \Xi_{N} .
$$

Concerning the pressure local space, we have the following natural choice for the basis:

$$
\mathbb{Q}_{N}^{k}=\operatorname{span}\left\{\psi_{i j}(x, y): \psi_{i j} \circ \boldsymbol{F}_{k}(\xi, \eta)=l_{i}(\xi) l_{j}(\eta), 1 \leq i, j \leq N-1\right\} .
$$

We can now express the local solutions in a given element $\Omega_{k}$ as

$$
\begin{aligned}
& \tilde{\boldsymbol{u}}_{N}^{k}(\xi, \eta)=\sum_{i=0}^{N} \sum_{j=0}^{N-1} \boldsymbol{u}_{i j}^{k} h_{i}(\xi) h_{j}(\eta)+\boldsymbol{u}_{0 N}^{k} h_{N}(\eta), \\
& \tilde{p}_{N}^{k}(\xi, \eta)=\sum_{i, j=1}^{N-1} p_{i j}^{k} l_{i}(\xi) l_{j}(\eta),
\end{aligned}
$$

where

$$
\begin{aligned}
& \boldsymbol{u}_{i j}^{k}=\boldsymbol{u}_{N} \circ \boldsymbol{F}_{k}\left(\xi_{i}, \xi_{j}\right), \quad i=0, \cdots, N, \quad j=0, \cdots, N-1 ; \\
& \boldsymbol{u}_{0 N}^{k}=\boldsymbol{u}_{N} \circ \boldsymbol{F}_{k}(0,1) ; \\
& p_{i j}^{k}=p_{N} \circ \boldsymbol{F}_{k}\left(\xi_{i}, \xi_{j}\right), \quad i, j=1, \cdots, N-1 .
\end{aligned}
$$

Plugging the above expansions into (3.2), and choose the nodal basis functions of $\boldsymbol{X}_{N}$ as 
test functions, we find that the term (3.4) becomes: for $m=0,1, \cdots, N ; n=0,1, \cdots, N-1$,

$$
\begin{aligned}
& \sum_{(i, j) \in \Xi_{N}} a_{m n, i j}^{k} u_{i j}^{k}=a_{N}^{k}\left(u_{N}^{k}, \varphi_{m n}\right) \\
& =\sum_{p, q=0}^{N}\left[G_{1}^{k}\left(\xi_{p}, \xi_{q}\right)\left(\sum_{i=0}^{N} \sum_{j=0}^{N-1} u_{i j}^{k} h_{i}^{\prime}\left(\xi_{p}\right) h_{j}\left(\xi_{q}\right)\right) h_{m}^{\prime}\left(\xi_{p}\right) h_{n}\left(\xi_{q}\right) \omega_{p q}\right] \\
& \quad+\sum_{p, q=0}^{N}\left[G_{2}^{k}\left(\xi_{p}, \xi_{q}\right)\left(\sum_{i=0}^{N} \sum_{j=0}^{N-1} u_{i j}^{k} h_{i}\left(\xi_{p}\right) h_{j}^{\prime}\left(\xi_{q}\right)+u_{0 N}^{k} h_{N}^{\prime}\left(\xi_{q}\right)\right) h_{m}\left(\xi_{p}\right) h_{n}^{\prime}\left(\xi_{q}\right) \omega_{p q}\right] \\
& \quad+\sum_{p, q=0}^{N}\left[G_{3}^{k}\left(\xi_{p}, \xi_{q}\right)\left(\sum_{i=0}^{N} \sum_{j=0}^{N-1} u_{i j}^{k} h_{i}^{\prime}\left(\xi_{p}\right) h_{j}\left(\xi_{q}\right)\right) h_{m}\left(\xi_{p}\right) h_{n}^{\prime}\left(\xi_{q}\right) \omega_{p q}\right] \\
& \quad+\sum_{p, q=0}^{N}\left[G_{3}^{k}\left(\xi_{p}, \xi_{q}\right)\left(\sum_{i=0}^{N} \sum_{j=0}^{N-1} u_{i j}^{k} h_{i}\left(\xi_{p}\right) h_{j}^{\prime}\left(\xi_{q}\right)+u_{0 N}^{k} h_{N}^{\prime}\left(\xi_{q}\right)\right) h_{m}^{\prime}\left(\xi_{p}\right) h_{n}\left(\xi_{q}\right) \omega_{p q}\right] \\
& =\sum_{p=0}^{N} G_{1}^{k}\left(\xi_{p}, \xi_{n}\right) D_{p m}\left(\sum_{i=0}^{N} D_{p i} u_{i n}^{k}\right) \omega_{p n}+\sum_{q=0}^{N} G_{2}^{k}\left(\xi_{m}, \xi_{q}\right) D_{q n}\left(\sum_{j=0}^{N-1} D_{q j} u_{m j}^{k}+D_{q N} u_{0 N}^{k}\right) \omega_{m q} \\
& \quad+\sum_{q=0}^{N-1} G_{3}^{k}\left(\xi_{m}, \xi_{q}\right) D_{q n}\left(\sum_{i=0}^{N} D_{m i} u_{i q}^{k}\right) \omega_{m q}+\sum_{p=0}^{N} G_{3}^{k}\left(\xi_{p}, \xi_{n}\right) D_{p m}\left(\sum_{j=0}^{N-1} D_{n j} u_{p j}^{k}+D_{n N} u_{0 N}^{k}\right) \omega_{p n},
\end{aligned}
$$

where $D_{k j}=h_{j}^{\prime}\left(\xi_{k}\right), G_{1}^{k}, G_{2}^{k}$ and $G_{3}^{k}$ are geometric factors in (3.5) related to the mapping (A.1).

For the basis function, $h_{N}(\eta)$, corresponding to the index $(m, n)=(0, N)$, noticing that the derivative of $h_{N}(\eta)$ with respect to $\tilde{\xi}$ vanishes, we have

$$
\begin{aligned}
& \sum_{(i, j) \in \Xi_{N}} a_{0 N, i j}^{k} u_{i j}^{k}=a_{N}^{k}\left(u_{N}^{k}, h_{N}(\eta)\right) \\
&= \sum_{p, q=0}^{N}\left[G_{2}^{k}\left(\xi_{p}, \xi_{q}\right)\left(\sum_{i=0}^{N} \sum_{j=0}^{N-1} u_{i j}^{k} h_{i}\left(\xi_{p}\right) h_{j}^{\prime}\left(\xi_{q}\right)+u_{0 N}^{k} h_{N}^{\prime}\left(\xi_{q}\right)\right) h_{N}^{\prime}\left(\xi_{q}\right) \omega_{p q}\right] \\
&+\sum_{p, q=0}^{N}\left[G_{3}^{k}\left(\xi_{p}, \xi_{q}\right)\left(\sum_{i=0}^{N} \sum_{j=0}^{N-1} u_{i j}^{k} h_{i}^{\prime}\left(\xi_{p}\right) h_{j}\left(\xi_{q}\right)\right) h_{N}^{\prime}\left(\xi_{q}\right) \omega_{p q}\right] \\
&=\sum_{p, q=0}^{N}\left[G_{2}^{k}\left(\xi_{p}, \xi_{q}\right)\left(\sum_{i=0}^{N} \sum_{j=0}^{N-1} u_{i j}^{k} \delta_{p i} D_{q j}+u_{0 N}^{k} D_{q N}\right) D_{q N} \omega_{p q}\right] \\
& \quad+\sum_{p, q=0}^{N}\left[G_{3}^{k}\left(\xi_{p}, \xi_{q}\right)\left(\sum_{i=0}^{N} \sum_{j=0}^{N-1} u_{i j}^{k} D_{p i} \delta_{q j}\right) D_{q N} \omega_{p q}\right] \\
&=\sum_{q=0}^{N}\left[\sum_{p=0}^{N} G_{2}^{k}\left(\xi_{p}, \xi_{q}\right) D_{q N}\left(\sum_{j=0}^{N-1} D_{q j} u_{p j}^{k}+D_{q N} u_{0 N}^{k}\right) \omega_{p q}\right] \\
& \quad+\sum_{q=0}^{N-1}\left[\sum_{p=0}^{N} G_{3}^{k}\left(\xi_{p}, \xi_{q}\right) D_{q N}\left(\sum_{i=0}^{N} D_{p i} u_{i q}^{k}\right) \omega_{p q}\right] .
\end{aligned}
$$


It is worthwhile to mention that in the last equality, the term corresponding to $q=N$ disappears due to the presence of the Kronecker symbol $\delta_{q j}$. We see that, despite the possible presence of singular Jacobian $J_{k}$ at the top side, the matrix-vector multiplication corresponding to $\left(\nabla \boldsymbol{u}_{N}, \nabla \boldsymbol{v}_{N}\right)_{N, \Omega_{k}}$ contains no singular term.

Obviously, the matrix-vector multiplication corresponding to $\left(p_{N}, \nabla \cdot v_{N}\right)_{N, \Omega_{k}}$, namely

$$
\sum_{i, j=1}^{N-1} \boldsymbol{d}_{m n, i j}^{k} p_{i j}^{k}, \quad m, n=1, \cdots, N-1
$$

can be treated in a similar fashion.

The global matrix system can be built by assembling the local matrix system, together with the continuity conditions for the velocity, which can be accomplished by requiring that the neighboring solutions share same nodal values at the interfaces. Finally the resulting set of algebraic equations can be written in a matrix form,

$$
\left\{\begin{array}{l}
\boldsymbol{A}_{N} \underline{\boldsymbol{u}}+\boldsymbol{D}_{N} \underline{p}=\boldsymbol{B}_{N} \underline{f} \\
\boldsymbol{D}_{N}^{T} \underline{\boldsymbol{u}}=0
\end{array}\right.
$$

or, in component form,

$$
\left\{\begin{array}{l}
\left.\sum_{k=1}^{K} \Gamma\left[\sum_{(i, j) \in \Xi_{N}^{k}} a_{m n, i j}^{k} \boldsymbol{u}_{i j}^{k}+\sum_{i, j=1}^{N-1} \boldsymbol{d}_{m n, i j}^{k} p_{i j}^{k}\right]=\sum_{k=1}^{K} \Gamma b_{m n}^{k}\right\}_{m n^{\prime}}^{k} \quad(m, n) \in \Xi_{N}^{k}, \\
\sum_{i, j=1}^{N-1} \boldsymbol{d}_{i j, m n}^{k} \boldsymbol{u}_{i j}^{k}=0, \quad m, n=1, \cdots, N-1 ; \quad k=1, \cdots, K,
\end{array}\right.
$$

where $\Xi_{N}^{k}$ is the set $\{(i, j) ; 0 \leq i, j \leq N\}$ if $\Omega_{k}$ is a quadrilateral or is defined by (3.6) if $\Omega_{k}$ is a triangle, and $\sum^{\Gamma}$ means the summation after taking into account the continuity of the velocity on the elemental interfaces and the Dirichlet boundary conditions on the velocity.

Using a block Gaussian elimination, this discrete spectral-element saddle problem (3.7) can be decoupled into two positive definite systems for the pressure and the velocity respectively, which are then solved by using a preconditioned conjugate gradient method. For more detail in this regard, we refer to [12,21].

\section{Numerical results}

We present in this section several numerical experiments to demonstrate the flexibility, accuracy as well as the shortcomings of the proposed unstructured spectral-element method. In particular, we demonstrate the applicability of this method for simulations of incompressible flows governed by the Navier-Stokes equations. 

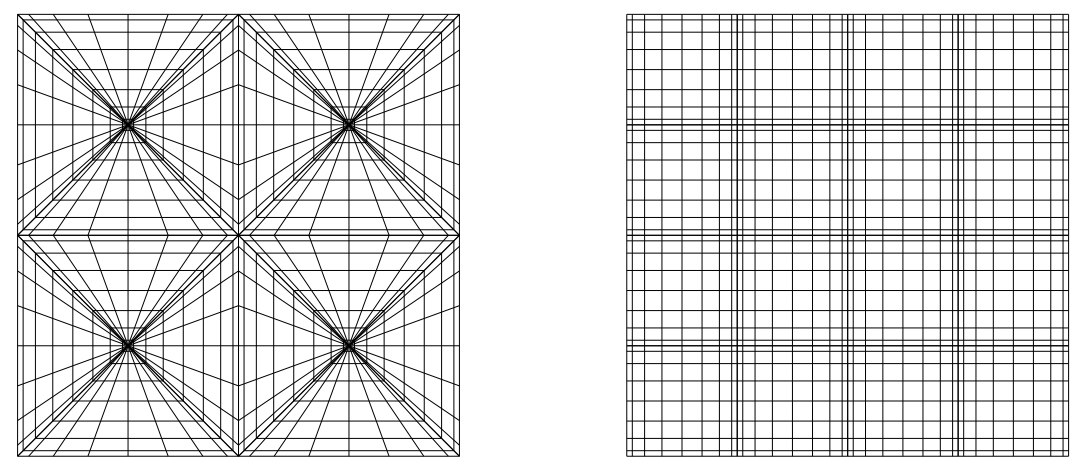

Figure 3: Triangular partition (left); Quadrangular partition (right).

\subsection{Accuracy and stability test}

We examine here the accuracy and stability of the triangular spectral-element method and compare them with the quadrangular spectral-element method.

We consider the Navier-Stokes equations

$$
\begin{cases}\frac{\partial u}{\partial t}+\boldsymbol{u} \cdot \nabla \boldsymbol{u}-v \Delta \boldsymbol{u}+\nabla p=f, & \text { in } \Omega \times(0, T), \\ \nabla \cdot \boldsymbol{u}=0, & \text { in } \Omega \times(0, T), \\ \boldsymbol{u}=\boldsymbol{g}, & \text { on } \partial \Omega \times(0, T), \\ \boldsymbol{u}=\boldsymbol{u}_{0}, & \text { on } \Omega \times\{0\} .\end{cases}
$$

The above equations are first discretized in time by using a second-order semi-implicit BDF-Adam-Bashforth scheme. This leads to, at each time step, a generalized Stokes problem which is then discretized by using the triangular and quadrangular spectral-element methods. The final linear system after the space discretization is solved by using an innerouter preconditioned conjugate gradient procedure combined with the Uzawa algorithm (cf. [12]).

We first solve the above equations with $v=1$ and with the steady state solution

$$
\begin{aligned}
& u(x, y, t)=(\sin x \cos y,-\cos x \sin y), \\
& p(x, y, t)=\sin x \sin y .
\end{aligned}
$$

In Fig. 4 , we plot, in a semi-log scale, the $L^{2}$-errors and the $H^{1}$-errors obtained from the triangular and quadrangular spectral-element methods with the partitions in Fig. 3. It is observed that the errors of the numerical solutions decay exponentially, and the convergence rates using the two partitions are comparable.

Next, we examine the effect of clustering of points near the singular vertex on the stability of a popular time discretization scheme in which the time derivative is approximated by the second-order BDF formula while the nonlinear convective terms are treated 

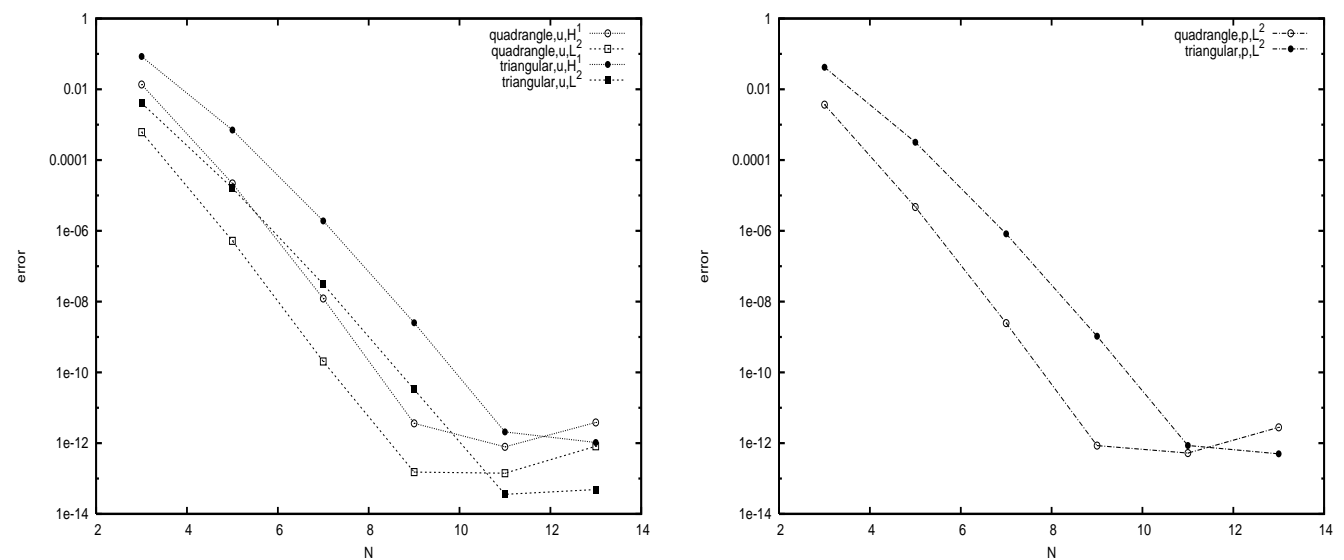

Figure 4: Errors in the $L^{2}$ - and $H^{1}$-norms versus the polynomial degree, obtained with the triangular and the quadrangular spectral-element methods. Left: velocity error; Right: pressure error.

in the frame of semi-Lagrange method [21]. In Table 1, we list the maximum allowable time steps for solving the Navier-Stokes equations (4.1) with $v=0.001$ using the quadrilateral and triangular partitions in Fig. 3. It is observed that the time steps required for the triangular spectral-element method are generally 2-3 times smaller than that for the corresponding quadrangular spectral-element method.

Table 1: Maximum allowable time steps for the quadrilateral and triangular partitions.

\begin{tabular}{||c|c|c|c|c|c|c||}
\hline$N$ & 4 & 6 & 8 & 10 & 12 & 14 \\
\hline$\triangle$ & $0.091-0.092$ & $0.044-0.045$ & $0.026-0.027$ & $0.016-0.017$ & $0.012-0.013$ & $0.0092-0.0093$ \\
\hline$\square$ & $0.15-0.16$ & $0.13-0.14$ & $0.078-0.079$ & $0.05-0.051$ & $0.035-0.036$ & $0.022-0.023$ \\
\hline
\end{tabular}

\subsection{Effects on conditioning}

While the use of nodal tensorial triangular elements offers the geometric flexibility, it does have a negative impact on the conditioning of the system matrix due to the fact that collocation points are densely clustered near the collapsed node (see Fig. 2).

Let $A_{N}$ be the system matrix associated with the Laplace operator using the quadrangular and triangular spectral-element partitions in Fig. 3, and $\boldsymbol{H}_{N}:=\beta I+\boldsymbol{A}_{N}$ We compare in Table 2 their condition numbers and growth rates $\alpha$ defined by

$$
\alpha_{C}=\log (\operatorname{cond}(C)) / \log N
$$

for a given matrix $C$. It is shown that the condition numbers of both $\boldsymbol{A}_{N}$ and $\boldsymbol{H}_{N}$ (with $\beta=1000$ ) in the triangular case grow much faster than the quadrangular case.

Next, we examine the condition numbers for the Uzawa operator $S_{N}:=\boldsymbol{D}_{N} \boldsymbol{A}_{N}^{-1} \boldsymbol{D}_{N}^{T}$ and the mass-matrix preconditioned Uzawa operator $\tilde{\boldsymbol{S}}_{N}:=\widetilde{\boldsymbol{B}}_{N}^{-1} \boldsymbol{S}_{N}$, where $\widetilde{\boldsymbol{B}}_{N}$ is the mass 
Table 2: Condition numbers for the generalized Helmholtz operator.

\begin{tabular}{||c|c|c|c|c|c|c|c|c||}
\hline & \multicolumn{3}{|c|}{$\triangle$} & \multicolumn{3}{|c||}{$\square$} \\
\hline$N$ & $\operatorname{cond}\left(\boldsymbol{A}_{N}\right)$ & $\alpha_{\boldsymbol{A}_{N}}$ & cond $\left(\boldsymbol{H}_{N}\right)$ & $\alpha_{\boldsymbol{H}_{N}}$ & $\operatorname{cond}\left(\boldsymbol{A}_{N}\right)$ & $\alpha_{\boldsymbol{A}_{N}}$ & $\operatorname{cond}\left(\boldsymbol{H}_{N}\right)$ & $\alpha_{\boldsymbol{H}_{N}}$ \\
\hline 4 & 270.8 & 4.04 & 63.7 & 3.00 & 58.2 & 2.93 & 32.5 & 2.51 \\
\hline 8 & 2319.3 & 3.73 & 356.4 & 2.83 & 218.9 & 2.59 & 49.5 & 1.88 \\
\hline 12 & 9959.7 & 3.70 & 2224.6 & 3.10 & 530.3 & 2.52 & 81.2 & 1.77 \\
\hline 16 & 30932.1 & 3.73 & 8104.2 & 3.24 & 1105.3 & 2.53 & 147.5 & 1.80 \\
\hline
\end{tabular}

Table 3: Condition numbers for the Uzawa operator.

\begin{tabular}{||c|c|c|c|c|c|c|c|c||}
\hline & \multicolumn{4}{|c|}{$\triangle$} & \multicolumn{4}{c||}{$\square$} \\
\hline$N$ & $\operatorname{cond}\left(\boldsymbol{S}_{N}\right)$ & $\alpha_{S_{N}}$ & $\operatorname{cond}\left(\widetilde{S}_{N}\right)$ & $\alpha_{\widetilde{S}_{N}}$ & $\operatorname{cond}\left(\boldsymbol{S}_{N}\right)$ & $\alpha_{S_{N}}$ & $\operatorname{cond}\left(\widetilde{S}_{N}\right)$ & $\alpha_{\widetilde{S}_{N}}$ \\
\hline 4 & 17.4 & 2.06 & 6.7 & 1.37 & 11.8 & 1.78 & 7.5 & 1.45 \\
\hline 8 & 29.7 & 2.73 & 8.7 & 1.04 & 57.0 & 1.94 & 8.6 & 1.0 \\
\hline 12 & 1616.2 & 2.97 & 9.3 & 0.90 & 146.5 & 2.01 & 9.2 & 0.89 \\
\hline 16 & 5384.7 & 3.10 & 9.6 & 0.82 & 286.5 & 2.04 & 9.6 & 0.82 \\
\hline
\end{tabular}

matrix associated to the Gauss quadrature. We observe in Table 3 that while the condition numbers of $S_{N}$ in the triangular case grows faster than that in the quadrangular case, their preconditioned matrices behave very similarly and only grow very slowly.

\subsection{Moffatt flow}

Next, we consider the Moffatt flow near a sharp corner at zero Reynolds number (Stokes flow). The computational domain is a triangle with three vertexes $(-1,0),(1,0),(0,-4)$. The domain is partitioned into 30 triangular elements (cf. left of Fig. 5) with $N=18$. The flow is driven by a tangential velocity $(1-x)(1+x)$ along the top of the domain. The streamline contours are plotted on the right of Fig. 5 . We are able to capture a cascade of
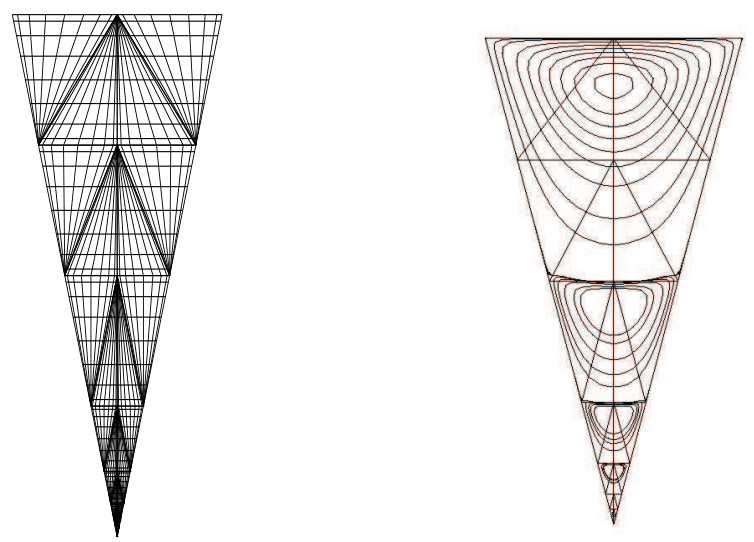

Figure 5: The domain partition (left), and the contours of the stream function (right). 
small eddies towards the bottom vertex. The results are in agreement with the Fig. 23 in paper [19].

\subsection{Flow through a backward facing step}

We now apply our spectral-element method to simulate the flow through a backward facing step by solving the Navier-Stokes equations (4.1) in the domain illustrated in Fig. 6.

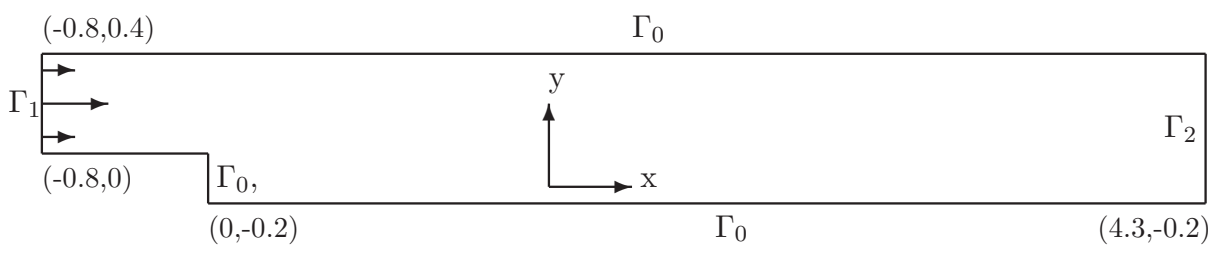

Figure 6: Domain configuration.

The boundary and initial conditions in (4.1) for the backward facing step flow are specified as follows:

$$
\begin{aligned}
& \boldsymbol{g}= \begin{cases}(0,0), & \text { on } \Gamma_{0}, \\
\left(1-(1-5 y)^{2}, 0\right), & \text { on } \Gamma_{1}, \\
\left(\frac{2}{3}\left[1-\left(1-\frac{10}{3}(y+0.2)\right)^{2}\right], 0\right), & \text { on } \Gamma_{2},\end{cases} \\
& \boldsymbol{u}_{0}=\left(\frac{2}{3}\left[1-\left(1-\frac{10}{3}(y+0.2)\right)^{2}\right], 0\right) .
\end{aligned}
$$

We consider the case of Reynolds number $\operatorname{Re}=\bar{u}(H-h) / v=191$, where $\bar{u}$ is the average velocity at the entrance, $H$ and $h$ are respectively the height of the outlet and the entrance. So $H-h$ means the height of the step. The expansion ratio of the step is $h: H=2: 3$. The profile of the inflow boundary condition is taken parabolic, as specified in (4.2). The outlet boundary is taken far away from the step (22 step heights) to avoid possible artificial reflection. The spectral-element meshes based on a pure quadrangular partition and a mixed triangular/quadrangular partition are shown in Fig. 7.

The Navier-Stokes equations (4.1) are first discretized in time by using a second order $\mathrm{BDF}$ scheme for the linear part, and the nonlinear convective terms are treated in the frame of semi-Lagrange method [21].

We used $N=8$ in each macro-element and the time step size $\Delta t=2 \times 10^{-4}$. All the presented results correspond to the numerical solution captured at $t=1.482$.

We validated the unstructured spectral-element method by comparing the results with those obtained by a structured rectangular spectral-element mesh. Figs. 8-10 show respectively the velocity vectors, pressure and streamline contours obtained by the quadrangular spectral-element method (top) and triangular spectral-element method (bottom). There is no noticeable difference between the two methods. 

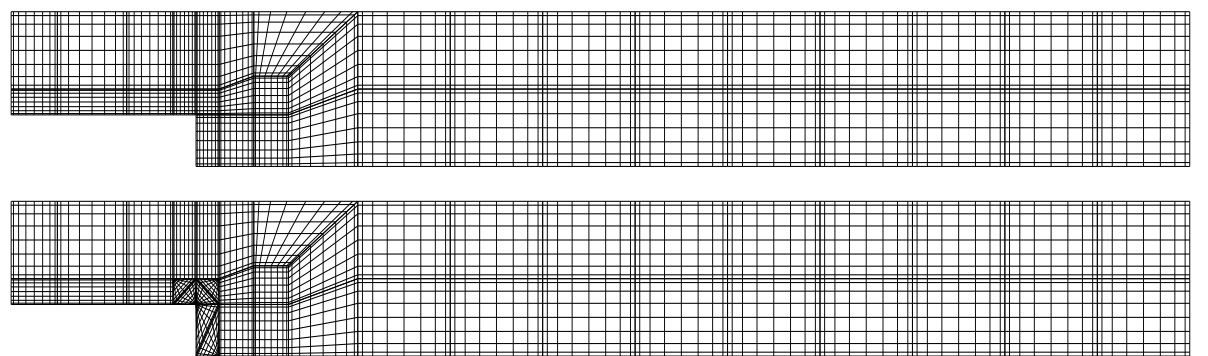

Figure 7: Domain partitions and spectral-element meshes for the backward facing step flow. The domain is broken into pure quadrangular elements (up) and mixed triangular/quadrangular elements (down).

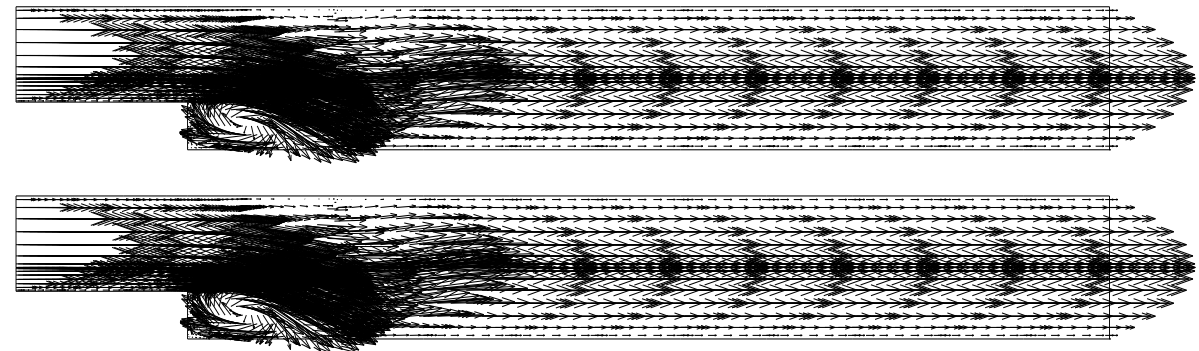

Figure 8: Instantaneous velocity vectors distribution. The results are essentially the same for the classical spectral-element method (up) and triangular spectral-element method (down).
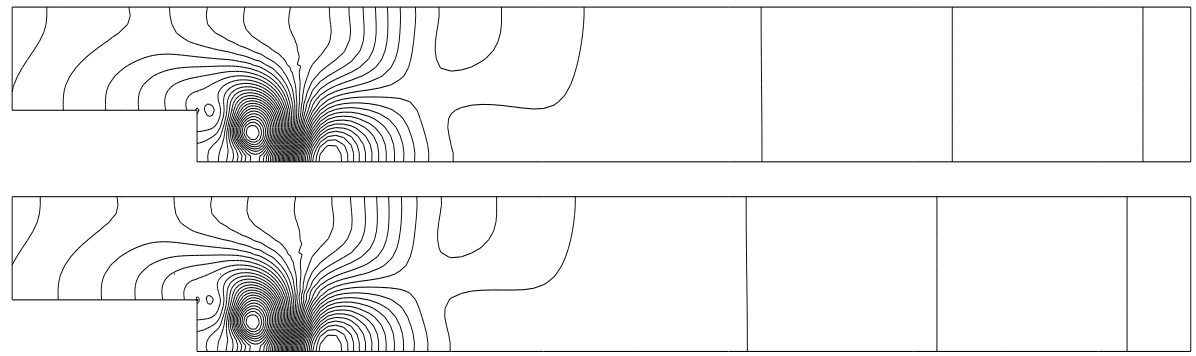

Figure 9: Pressure contours obtained respectively from the quadrangular spectral-element method (up) and triangular spectral-element method (down).
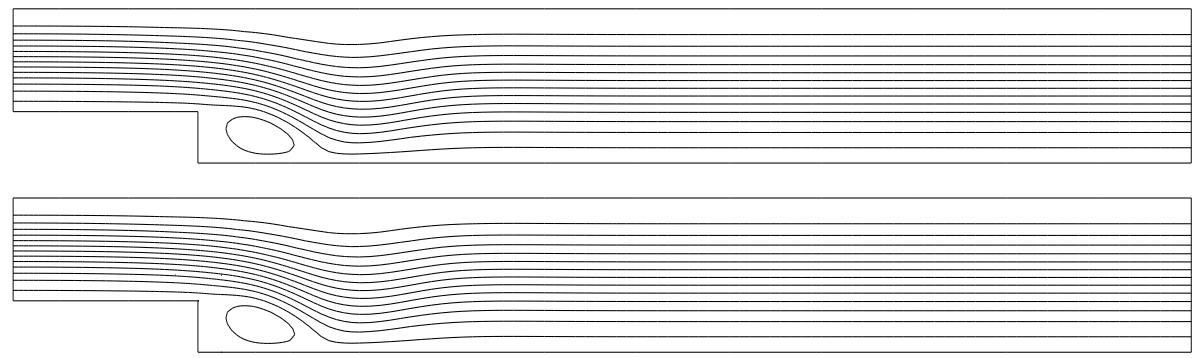

Figure 10: Streamline contours: quadrangular spectral-element method (up) and triangular spectral-element method (down). 

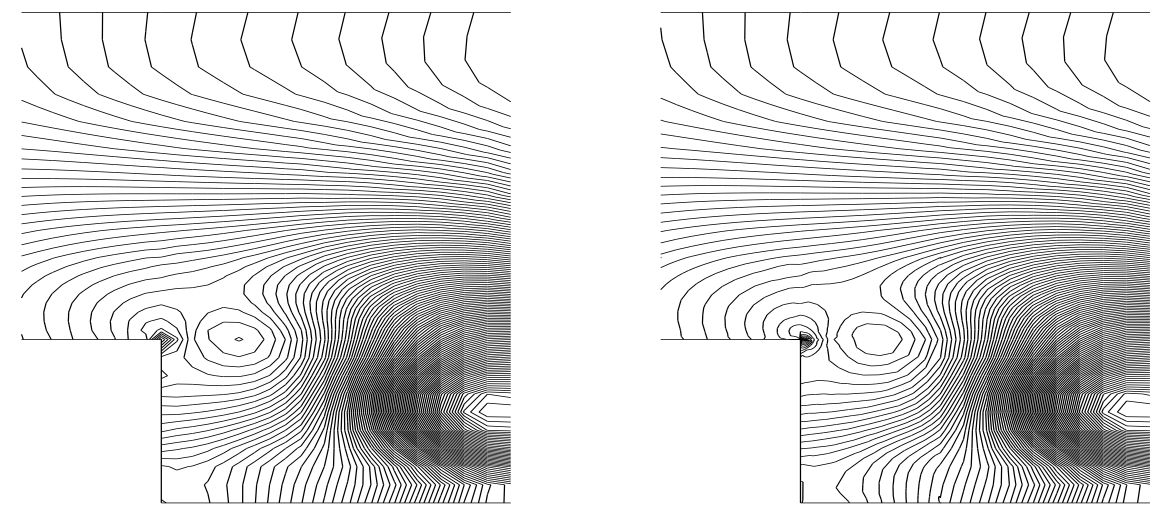

Figure 11: Enlarged pressure contourlines in the vicinity of the step: quadrangular spectral element method (left); triangular spectral-element method (right).

In order to make a more detailed comparison, we present in Fig. 11 an enlarged view of the pressure pattern near the step. It is observed that the unstructured mesh produces smoother pressure fields than the structured one. This is due to the fact that there are more grid points around the step corner in the unstructured mesh. This indicates that the clustering of the grid points near the "collapsed" vertex in the triangular elements is not always wasteful and can be useful in resolving corner singularities and sharp layers.

We observe that the triangular spectral method does lead to larger condition numbers and require more CG iterations as expected. However, it is worthwhile to mention that in most cases only a few triangular elements are needed to resolve the geometric singularity and allow geometric flexibility. For instance, in the backward-facing step example (cf. Fig. 7) considered above, only six triangular elements are needed near the re-entry corner. Hence, it is expected that the effect of the triangular elements on the assembled system matrices will not be as pronounced as in the single element case. This is confirmed by the results in Table 4 where we list the numbers of the outer Uzawa iterations for the Stokes problem and the number of inner CG iterations (for a tolerance of $10^{-8}$ ) for solving the two velocity components with the two meshes in Fig. 7.

Table 4: Condition numbers and iteration numbers of the Stokes solver in the backward-facing step flow.

\begin{tabular}{||c|c|c|c|c||}
\hline & $\begin{array}{c}\text { condition \# of } \\
\text { Uzawa operator }\end{array}$ & $\begin{array}{c}\text { \# of outer } \\
\text { Uzawa iterations }\end{array}$ & $\begin{array}{c}\text { \# of inner } \\
\text { iterations for } u_{1}\end{array}$ & $\begin{array}{c}\text { \# of inner } \\
\text { iterations for } u_{2}\end{array}$ \\
\hline$\triangle$ & 155.9 & 42 & 265 & 236 \\
\hline$\square$ & 152.7 & 41 & 194 & 176 \\
\hline
\end{tabular}

Note that we have only considered the straightforward diagonal and mass-matrix preconditioners here, it is expected that condition numbers and iteration numbers can be reduced significantly by using a low-order finite-element method on the same grid as a preconditioner (cf. [4,13]). However, how to efficiently solve the finite-element system on a spectral-element mesh which is not shape regular requires further investigation. 


\section{Conclusions}

We presented an unstructured nodal spectral-element method for the two-dimensional Stokes and Navier-Stokes equations. The method is based on a rational approximation in triangle/tetrahedrons and an easy-to-implement nodal basis. The main advantages of the proposed method are: (i) flexibility in handling complex domains; (ii) full tensor product property; (iii) an easy-to-implement nodal basis; and (iv) it can be easily extended to the three-dimensional case.

We presented several two-dimensional numerical experiments which demonstrated the flexibility and accuracy of the method for a variety of problems including incompressible flows governed by the time dependent Navier-Stokes equations.

It is also observed that the use of triangular elements leads to larger condition numbers and requires more iterations when a simple CG iteration with diagonal preconditioner is used. This loss in computational efficiency is the price we pay for the geometric flexibility. On the other hand, the loss can be significantly reduced if only a few necessary triangular elements are used to resolve the geometric singularity as in the backward facing step example. However, for full unstructured grid, it becomes important to construct a more efficient preconditioner by using perhaps a finite difference or finite element method on the same grid (cf. $[4,13])$. We shall leave this and the extension to three dimensional tetrahedral cases to a future work.

\section{Acknowledgments}

The work of the second author is partially supported by NFS grant DMS-0610646. The research of the third author was partially supported by National NSF of China (Grant number 11071203).

\section{Appendix: Computation of the geometric factors $G_{i}^{k}$}

Let the three vertexes of $\Omega_{k}$ be $\left(x_{1}, y_{1}\right),\left(x_{2}, y_{2}\right)$, and $\left(x_{3}, y_{3}\right)$. Then, the one-to-one transformation from $\square$ to $\Omega_{k}$ reads

$$
\left\{\begin{array}{l}
x=\frac{1}{4}(1+\xi)(1-\eta)\left(x_{2}-x_{1}\right)+\frac{1}{2}(1+\eta)\left(x_{3}-x_{1}\right)+x_{1}, \\
y=\frac{1}{4}(1+\xi)(1-\eta)\left(y_{2}-y_{1}\right)+\frac{1}{2}(1+\eta)\left(y_{3}-y_{1}\right)+y_{1},
\end{array} \quad \forall(\xi, \eta) \in \square,\right.
$$

with the inverse transformation

$$
\left\{\begin{array}{l}
\xi=2 \frac{\left(x-x_{1}\right)\left(y_{3}-y_{1}\right)-\left(x_{3}-x_{1}\right)\left(y-y_{1}\right)}{\left(x-x_{3}\right)\left(y_{2}-y_{1}\right)-\left(x_{2}-x_{1}\right)\left(y-y_{3}\right)}-1, \\
\eta=2 \frac{\left(x-x_{1}\right)\left(y_{2}-y_{1}\right)-\left(x_{2}-x_{1}\right)\left(y-y_{1}\right)}{\left(x_{3}-x_{1}\right)\left(y_{2}-y_{1}\right)-\left(x_{2}-x_{1}\right)\left(y_{3}-y_{1}\right)}-1,
\end{array} \quad \forall(x, y) \in \Omega_{k} .\right.
$$


In addition, we can easily derive the following formulas:

$$
\begin{aligned}
& \frac{\partial \xi}{\partial x}=2 \frac{\left[\left(x_{3}-x_{1}\right)\left(y_{2}-y_{1}\right)-\left(x_{2}-x_{1}\right)\left(y_{3}-y_{1}\right)\right]\left(y-y_{3}\right)}{\left[\left(x-x_{3}\right)\left(y_{2}-y_{1}\right)-\left(x_{2}-x_{1}\right)\left(y-y_{3}\right)\right]^{2}}, \\
& \frac{\partial \xi}{\partial y}=-2 \frac{\left[\left(x_{3}-x_{1}\right)\left(y_{2}-y_{1}\right)-\left(x_{2}-x_{1}\right)\left(y_{3}-y_{1}\right)\right]\left(x-x_{3}\right)}{\left[\left(x-x_{3}\right)\left(y_{2}-y_{1}\right)-\left(x_{2}-x_{1}\right)\left(y-y_{3}\right)\right]^{2}} \\
& \frac{\partial \eta}{\partial x}=2 \frac{y_{2}-y_{1}}{\left(x_{3}-x_{1}\right)\left(y_{2}-y_{1}\right)-\left(x_{2}-x_{1}\right)\left(y_{3}-y_{1}\right)} \\
& \frac{\partial \eta}{\partial y}=-2 \frac{x_{2}-x_{1}}{\left(x_{3}-x_{1}\right)\left(y_{2}-y_{1}\right)-\left(x_{2}-x_{1}\right)\left(y_{3}-y_{1}\right)} \\
& \frac{\partial x}{\partial \xi}=\left(x_{2}-x_{1}\right) \frac{1-\eta}{4}, \quad \frac{\partial x}{\partial \eta}=-\left(x_{2}-x_{1}\right) \frac{1+\xi}{4}+\frac{1}{2}\left(x_{3}-x_{1}\right) \\
& \frac{\partial y}{\partial \xi}=\left(y_{2}-y_{1}\right) \frac{1-\eta}{4}, \quad \frac{\partial y}{\partial \eta}=-\left(y_{2}-y_{1}\right) \frac{1+\xi}{4}+\frac{1}{2}\left(y_{3}-y_{1}\right) \\
& J_{k}=\operatorname{det}\left(\frac{\partial(x, y)}{\partial(\xi, \eta)}\right)=\frac{1-\eta}{8}\left[\left(x_{2}-x_{1}\right)\left(y_{3}-y_{1}\right)-\left(x_{3}-x_{1}\right)\left(y_{2}-y_{1}\right)\right] .
\end{aligned}
$$

In virtue of above formulas, we obtain a relationship between $\nabla_{x} \varphi(x, y)$ and $\nabla_{\xi} \widetilde{\varphi}(\widetilde{\zeta}, \eta)$ as follows:

$$
\begin{aligned}
& \frac{\partial \varphi}{\partial x}= 2 \frac{\left[\left(x_{3}-x_{1}\right)\left(y_{2}-y_{1}\right)-\left(x_{2}-x_{1}\right)\left(y_{3}-y_{1}\right)\right]\left(y-y_{3}\right)}{\left[\left(x-x_{3}\right)\left(y_{2}-y_{1}\right)-\left(x_{2}-x_{1}\right)\left(y-y_{3}\right)\right]^{2}} \frac{\partial \varphi}{\partial \xi} \\
&+2 \frac{y_{2}-y_{1}}{\left(x_{3}-x_{1}\right)\left(y_{2}-y_{1}\right)-\left(x_{2}-x_{1}\right)\left(y_{3}-y_{1}\right)} \frac{\partial \varphi}{\partial \eta} \\
& \frac{\partial \varphi}{\partial y}=- \frac{\left[\left(x_{3}-x_{1}\right)\left(y_{2}-y_{1}\right)-\left(x_{2}-x_{1}\right)\left(y_{3}-y_{1}\right)\right]\left(x-x_{3}\right)}{\left[\left(x-x_{3}\right)\left(y_{2}-y_{1}\right)-\left(x_{2}-x_{1}\right)\left(y-y_{3}\right)\right]^{2}} \frac{\partial \varphi}{\partial \xi} \\
&-2 \frac{\partial \varphi}{\left(x_{3}-x_{1}\right)\left(y_{2}-y_{1}\right)-\left(x_{2}-x_{1}\right)\left(y_{3}-y_{1}\right)} \frac{\partial \eta}{\partial \eta} \\
& \frac{\partial \widetilde{\varphi}}{\partial \xi}=\left(x_{2}-x_{1}\right) \frac{1-\eta}{4} \frac{\partial \varphi}{\partial x}+\left(y_{2}-y_{1}\right) \frac{1-\eta}{4} \frac{\partial \varphi}{\partial y}, \\
& \frac{\partial \widetilde{\varphi}}{\partial \eta}=\left[-\left(x_{2}-x_{1}\right) \frac{1+\xi}{4}+\frac{1}{2}\left(x_{3}-x_{1}\right)\right] \frac{\partial \varphi}{\partial x}+\left[-\left(y_{2}-y_{1}\right) \frac{1+\xi}{4}+\frac{1}{2}\left(y_{3}-y_{1}\right)\right] \frac{\partial \varphi}{\partial y} .
\end{aligned}
$$

\section{References}

[1] J. P. Boyd. Chebyshev and Fourier spectral methods. Dover Publications Inc., Mineola, NY, second edition, 2001.

[2] D. Braess and C. Schwab. Approximation on simplices with respect to weighted Sobolev norms. J. Approx. Theory, 103(2):329-337, 2000. 
[3] L. Chen, J. Shen, and C. Xu. A triangular spectral method for the Stokes equations. Numer. Math.: Theory, Methods and Applications, to appear.

[4] M. O. Deville and E. H. Mund. Chebyshev pseudospectral solution of second-order elliptic equations with finite element preconditioning. J. Comput. Phys., 60:517-533, 1985.

[5] M. Dubiner. Spectral methods on triangles and other domains. J. Sci. Comput., 6(4):345-390, 1991.

[6] W. J. Gordon and C. A. Hall. Transfinite element methods: blending-function interpolation over arbitrary curved element domains. Numer. Math., 21:109-129, 1973/74.

[7] W. Heinrichs and B. I. Loch. Spectral schemes on triangular elements. J. Comput. Phys., 173(1):279-301, 2001.

[8] J. S. Hesthaven. From electrostatics to almost optimal nodal sets for polynomial interpolation in a simplex. SIAM J. Numer. Anal., 35(2):655-676 (electronic), 1998.

[9] G. E. Karniadakis and S. J. Sherwin. Spectral/hp element methods for CFD. Numerical Mathematics and Scientific Computation. Oxford University Press, New York, 1999.

[10] T. Koornwinder. Two-variable analogues of the classical orthogonal polynomials. In Theory and application of special functions (Proc. Advanced Sem., Math. Res. Center, Univ. Wisconsin, Madison, Wis., 1975), pages 435-495. Math. Res. Center, Univ. Wisconsin, Publ. No. 35. Academic Press, New York, 1975.

[11] Y. Maday and A. T. Patera. Spectral element methods for the incompressible Navier-Stokes equations. In IN: State-of-the-art surveys on computational mechanics (A90-47176 21-64). New York, American Society of Mechanical Engineers, 1989, p. 71-143. Research supported by DARPA., volume 1, pages 71-143, 1989.

[12] Y. Maday, D. Meiron, A. T. Patera, and E. M. Rønquist. Analysis of iterative methods for the steady and unsteady Stokes problem: application to spectral element discretizations. SIAM J. Sci. Comput., 14(2):310-337, 1993.

[13] S. A. Orszag. Spectral methods for complex geometries. J. Comput. Phys., 37:70-92, 1980.

[14] R. G. Owens. Spectral approximations on the triangle. R. Soc. Lond. Proc. Ser. A Math. Phys. Eng. Sci., 454(1971):857-872, 1998.

[15] R. Pasquetti and F. Rapetti. Spectral element methods on triangles and quadrilaterals: comparisons and applications. Journal of Computational Physics, 198(1):349-362, 2004.

[16] R. Pasquetti and F. Rapetti. Spectral element methods on unstructured meshes: comparisons and recent advances. J. Sci. Comput., 27(1-3):377-387, 2006.

[17] A. T. Patera. A spectral element method for fluid dynamics: laminar flow in a channel expansion. Journal of Computational Physics, 54(3):468-488, 1984.

[18] J. Shen, L.-L. Wang, and H. Li. A triangular spectral element method using fully tensorial rational basis functions. SIAM J. Numer. Anal., 47(3):1619-1650, 2009.

[19] S. J. Sherwin and G. E. Karniadakis. A triangular spectral element method; applications to the incompressible Navier-Stokes equations. Comput. Methods Appl. Mech. Engrg., 123(14):189-229, 1995.

[20] M. A. Taylor, B. A. Wingate, and R. E. Vincent. An algorithm for computing Fekete points in the triangle. SIAM J. Numer. Anal., 38(5):1707-1720 (electronic), 2000.

[21] C. Xu and R. Pasquetti. On the efficiency of semi-implicit and semi-Lagrangian spectral methods for the calculation of incompressible flows. International Journal for Numerical Methods in Fluids, 35(3):319-340, 2001.

[22] C. Xu and Y. Maday. A spectral element method for the time-dependent two-dimensional euler equations: applications to flow simulations. J. Comput. Appl. Math., 91(1):63-85, 1998. 